\title{
Exploration on the Management Method of Low Frequency Load Shedding in Regional Power Grid
}

\author{
Ren Guowei ${ }^{1} \quad$ Ren Rui ${ }^{2}$ \\ ${ }^{1}$ State Grid Xinyang power supply company, Xinyang, Henan, 464000, China \\ ${ }^{2}$ State Grid Luoshan power supply company, Luoshan, Henan,464200, China
}

Key words: power system; low frequency load-shedding; Exploration ;

\begin{abstract}
Based on low frequency load-shedding practice experiments of Xinyang power supply company, work principles as 'three reports, one examination and a table modification' is proposed to improve the management of power grid in substitute district. The methods introduced in the paper is proved to be effective in the practice.
\end{abstract}

\section{Introduction}

With the UHV power grid of Henan is greatly improved, the operation of the electric properties is more complex and therefore as an important means of effective prevention of power system risk, improve the level of operation and management of low frequency automatic reducing load device becomes more and more important. Low frequency load-shedding is one of the manners that inhibit the system frequency decrease, keep the system frequency stable and ensure a safe system operation, an $d$ it takes an irreplaceable role in power system stability and safe operation ${ }^{[1,2,3]}$. In present low frequency load-shedding strategy designed and used by regional power grid, five basic turns are set to prevent the frequency drop when the active power shortage occurs in the system, and another three special turns are used to solve the problems that the frequency is still below $49.5 \mathrm{~Hz}$ during its recovery process. The capacity of low frequency load-shedding is required to reach $35 \% \sim 40 \%$ of the average maximal capacity of the local area. The disconnected capacity is distributed into thousands of lines connected to hundreds of 35 110 kV transformer substations. Among the disconnected capacity, the capacity related to the power companies in the factored county is similar to $60 \%$ of the regional power supply, and the setting of disconnected capacity in the strategy is more than $60 \%$ of the low frequency load-shedding in regional power network. How to make sure the disconnected capacity in each turn meets the requirements, especially ensure the amount of disconnected capacity in the county, is the key measure avoiding the system frequency collapse and blackouts caused by system faults.

The general management framework of low frequency load-shedding in regional power system is that the power dispatch department determines the operation modes in the next year based on the policy in the coming year of the low frequency load-shedding strategy, the demanded disconnected capacity in each turn and the possible maximal active power shortage, provided by the province power company, and delivers the final planning to each department by documents. The low frequency scheme of the substation is presented in a sheet with detailed tables and values, which includes the action frequency, the action time and the lock condition; the factored power company is only required to cut off the loads in turn. Strategy implementation: the urban substation should offer the completed sheets of low frequency load-shedding to the regional power dispatch department. The factored power company submits the reports of low frequency load-shedding to the regional power dispatch department for putting on file. During the normal operation, the disconnected capacity, its turns and the detailed lines are displayed in the dispatch station by dispatching technology support system; the factored power company provides the table of low frequency load-shedding on typical days.

\section{Methodology}

The problems in the management of low frequency load-shedding: the power dispatch department 
in urban power company participates the strategy of low frequency load-shedding in regional power grid and all real-time system states can be monitored, controlled and in regional working station, so the substation of urban power company is able to satisfy the scheme requirements in the control of load-shedding. However, the implementation of the strategy in rural power company is not optimistic, and several reasons can be drawn from the historical statistics:

(1) In view of the policy, the management is loose and most county power companies are factored companies. In view of the management process, the expected scheme requires the detailed action frequency of load-shedding, the action time, disconnected capacity, completed time and the report sheets in each month.

(2) The detection device for low frequency equipment is backward, and there is even no special detection devices. Problems exist fundamentally in scheme implementation.

(3) The recognition of the importance of low frequency load-shedding equipment lacks, and the attitude of scheme implementation is negative due to the thoughts that the equipment leads to power limitation.

(4) The load-shedding equipment is seriously aged, and the investment and replacement is not in time.

(5) The personnel using the equipment lack experiences and the implementation of project detection is not completed and accurate.

(6) The transfer method of low frequency load-shedding equipment is not managed well. The reports show everything is implemented correctly, but actually the equipment circuit loop is in a wrong connectivity. For example, in a special project detection for the utilization of low frequency load-shedding equipment, one third of the load-shedding turns in substation cannot act, the reason is: the detection and transfer reports provided by staffs are correct; the equipment transfer is verified by inquiring the staffs; the equipment circuit is open from the output terminal and low frequency components is added on the output terminal for equipment test, then the action values during the test process is correct and the voltage measurement is detected on the low frequency break terminal. After the test, the system circuit is recovered in normal condition. Through the expert investigation, it is found that is circuit break is due to the metal oxidization on the equipment terminal and the screw is not tighten. The ultimate cause is due to the incorrect transfer approach.

(7)Since the grade difference between two neighbored turns is small and the frequency difference is $0.25 \mathrm{~Hz}$ while the action time is $0.15 \mathrm{~s}$, each turn is related to many substations, the lines may be disconnected skipping grades if the error adjusting is controlled inconsistently.

(8)The value of disconnected capacity is a dynamic and the ratio of the disconnected capacity to the system capacity is almost constant. However, seen from the monthly report sheets, the ratio values are different in each season and cannot satisfy the scheme requirements. The directors of the factored companies cannot make sure whether the disconnected capacity meets the regional requirements.

The strategy corresponding to problems happened in the management of low frequency load-shedding in factored companies.

By analyzing the reason corresponding to above stated problems, it obtains: the problem of a true implementation and conduction of low frequency load-shedding scheme is the weak section in the entire management. So, what management approach should be used to obtain the true implementation?

Method 1: utilizing the urban detection groups to take in charge of the low frequency load-shedding scheme in factored power company.

Method 2: the urban power company organizes the technical training independently, and the unified check and acceptance is conducted by the urban power company.

Method 3 The urban power company organizes the technical training independently and change the management methods, then spot check is carried out by the urban power company.

Adopting method 1, it can efficiently solve 7 problems among the 8 problems stated above. For the unsolvable problem: the disconnected capacity of load-shedding is dynamic, and the ratio of the disconnected capacity to the system capacity is technically required to be constant; and seen from 
the monthly report sheets, the ratio values are different in each season and cannot satisfy the scheme requirements. In face of the situation that lots of substations and connected lines are widely distributed, the realization from the view of staffs, devices and time is difficult.

Adopting method 2, through technical training, it can help the technical and managing staffs in rural power company improve the knowledge about low frequency load shedding, however, the problem of equipment detection is still difficult and the detailed implementation situation is hard to control.

Adopting method 3, it can solve the problem in method 2 efficiently, the detailed procedure is: organize the technical training and invite the designing staffs, operation staffs, the staffs in secondary devices in production department and detection staffs to study together, the training contents include:

(1)The base and the detailed requirements of the low frequency load-shedding importance and the strategy.

(2)Invite the experts in low frequency load-shedding equipment detection to give lectures about the equipment detection, the transfer method and the detailed system circuit.

(3)Invite the manufacturers to present the method of the equipment and the detection tools, and let each power company practice once in order to find and rectify problems, and finally let all participants in this training manage the whole process of low frequency load-shedding implementation strategy.

Arrange the low frequency load-shedding implementation time schedule for each rural power company, then regulate and adjust the equipment among all regional and rural power companies to solve the problems in rural power companies and meet the requirements.

\section{Management}

Encourage management method innovation in order to efficiently control the design, detection, transfer implementation and capacity demands, and the detailed approach is "three reports, one examination and a table modification”. The three reports are: the original reports of low frequency load-shedding scheme and its implementation is changed into the reports of low frequency load-shedding scheme, implementation and the equipment detection; the transfer report over the equipment detection includes the transfer method, the transfer connection and transfer data.

One examination is to take a temporary spot check in the transformer substation, Verifying whether the switch action of low frequency load-shedding is correct, whether the actual adjusting value is consistent with the reports and whether the disconnected capacity meets the demands, and inquiring the protection staffs about the transfer details. Then let the staffs transfer once and check whether it is correct. One table modification is to modify the low frequency load-shedding table on typical days by changing Table 2 into Table 1. The added contents consist of the disconnected capacity percentage of the urban low frequency load-shedding scheme and the error of ratio between the actual disconnected capacity and the demanded capacity. The modified summary sheet is able to help the corresponding staffs understand whether the actual disconnected capacity satisfies the urban company requirements, and overcome the problems that the original sheet cannot clearly and efficiently present the ratio of the capacity disconnected in each turn to the regional network capacity and the ratio requirement given by the urban company.

\section{Conclusions}

The disconnected capacity is distributed into thousands of lines connected to hundreds of transformer substations. Among the disconnected capacity, the capacity related to the power companies in the factored power companies is similar to $60 \%$ of the regional power supply, and each single line participating in low frequency load-shedding has small capacity. Through the method of "three reports, one examination and a table modification", the problems, which include the improper implementation of the schemes, incorrect transferring method, the inefficient reports that cannot let the managers in each level realize whether the disconnected capacity meets the 
demanded values, are efficiently solved.

Table 1 Changed Low Frequency Load-shedding Summary Sheet

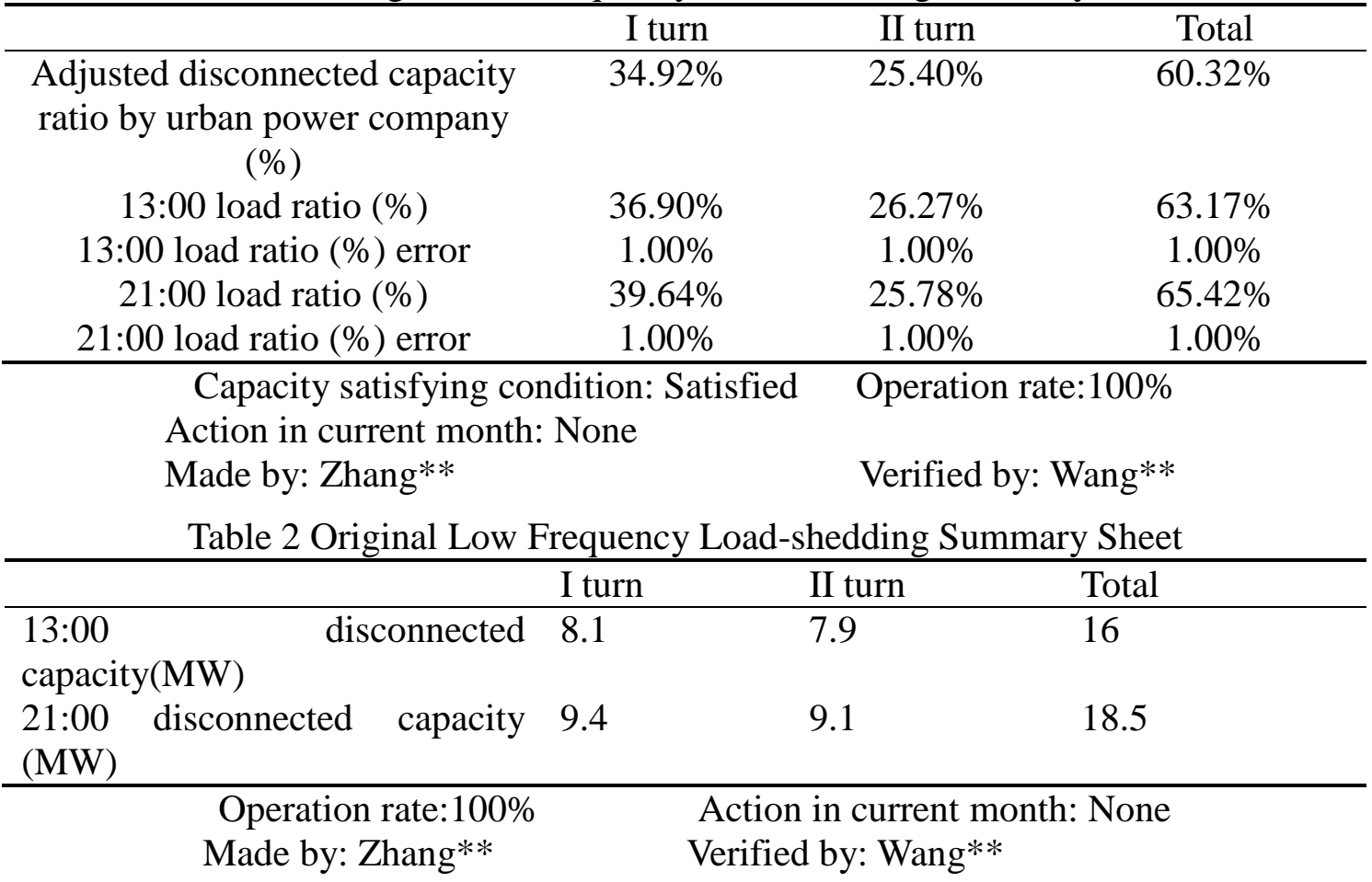

\section{References}

[1] Weiping Tian, Jianshe Bi. A Coordinated Load Shedding Scheme for Both Under voltage and Under frequency Load Shedding [J]. Electric Power Science and Engineering. 2005,2(10): 12-15

[2] Yongjian Cui. Research on Computer Load Shedding Technology [D], Chongqing University, 2001

[3] Hongbin Li. Application and enhancement of under frequency load shedding devices[J]. Hunnan Power Supply, 2001.2(2):18-22

[4]LU Zongxiang WANG Caixia, MIN Yong, ZHOU Shuangxi, Lü Jinxiang, WANG Yunbo. Summary of micro grid research[J]. Automation of electric power system. 2007,31(19):100-107 